\section{Fatores associados à obesidade global e à obesidade abdominal em mulheres na pós-menopausa}

\section{Factors associated with body and abdominal obesity in post-menopausal women}

\author{
Ana Paula França 1 \\ José Mendes Aldrighi 2 \\ Maria de Fátima Nunes Marucci 3
}

1 Programa Interunidades em Nutrição Humana AplicadaPRONUT. Av. Prof. Lineu Prestes, 580, Bloco 13A. Cidade Universitária. São Paulo, SP, Brasil. CEP: 05.508-000. E-mail: apfranca@usp.br

2 Departamento de Saúde Materno Infantil. Faculdade de Saúde Pública. Universidade de São Paulo. São Paulo, SP, Brasil. 3 Departamento de Nutrição. Faculdade de Saúde Pública. Universidade de São Paulo. São Paulo, SP, Brasil.

\begin{abstract}
Objectives: to evaluate the body and abdominal obesity in post-menopausal women, according to educational level, level of physical activity, use of hormone therapy (HT), and parity.

Methods: 157 post-menopausal women, attending two public out-patients clinics in the city of São Paulo, State of São Paulo, Brazil, were interviewed. The prevalence of body obesity was calculated using the body mass index $(B M I \geq 30.0)$ and the body fat percentage $(\% B F \geq 37 \%)$. Abdominal obesity was calculated using the waist hip ratio (WHR $\geq 0.85)$.

Results: the prevalence of body obesity was $34.4 \%$ (using the BMI) and 40.1\% (using \%BF). The prevalence of abdominal obesity was $73.8 \%$. Most of the women had up to seven years of formal schooling (47.8\%), were considered sedentary or insufficiently active (52.3\%), and had never used oral HT or had been using it for less than 12 months (72.0\%). A higher prevalence of body obesity was found women who engaged in little physical activity and did not use $H T(p<0.05)$. As for abdominal obesity, a risk factor for cardiovascular disease (CVD), only the level of education was associated with WHR $(p<0.05)$.

Conclusions: post-menopausal women who are physically active and use HT showed a lower prevalence of body obesity, although the prevalence of abdominal obesity was still high and higher still among those with a lower level of education.
\end{abstract}

Key words Menopause, Global obesity, Abdominal obesity

\section{Resumo}

Objetivos: avaliar a prevalência de obesidade global e obesidade abdominal, em mulheres pós-menopausa, segundo o grau de instrução, nivel de atividade física, uso de terapia hormonal na menopausa (THM) e paridade.

Métodos: foram entrevistadas 157 mulheres na pósmenopausa, atendidas em dois ambulatórios públicos da cidade de São Paulo, São Paulo, Brasil. A obesidade foi determinada segundo o indice de massa corporal (IMC $\geq 30,0)$ e segundo o percentual de gordura corporal $(\% G C \geq 37 \%)$. A obesidade abdominal foi determinada pela relação cintura-quadril $(R C Q \geq 0,85)$.

Resultados: a prevalência de obesidade global foi $34,4 \%$ (segundo o IMC) e de 40,1\% (segundo o \%GC). A prevalência de obesidade abdominal foi de 73,8\%. Grande parte das entrevistadas referiu até sete anos de instrução formal (47,8\%), foi considerada sedentária ou insuficientemente ativa (52,3\%) e nunca tinha utilizado THM oral ou tinha utilizado por menos de 12 meses (72,0\%). Foi constatada maior prevalência de obesidade global no grupo de mulheres sedentárias ou insuficientemente ativas e no grupo de não usuárias de THM $(p<0,05)$. Quanto à obesidade abdominal, fator de risco para doenças cardiovasculares (DCV), apenas o grau de instrução mostrou-se associado à $R C Q(p<0,05)$.

Conclusões: mulheres após a menopausa, fisicamente ativas e usuárias de THM exibiram menor prevalência de obesidade global, porém a prevalência de obesidade abdominal foi alta e ainda maior nas mulheres com menor grau de instrução.

Palavras-chave Menopausa, Obesidade global, Obesidade abdominal 


\section{Introdução}

A obesidade é uma complexa afecção crônica, resultante da interação de fatores genéticos e do meio ambiente e, na mulher, relaciona-se à infertilidade, depressão, distúrbios alimentares, baixa auto-estima, 1 hipertensão, dislipidemias, infarto, diabetes tipo 2, doença cardiovascular (DCV), câncer de mama, de endométrio e de cólon intestinal.1,2

Após os 50 anos a mulher apresenta tendência ao aumento de peso, que pode estar relacionada à redução das necessidades energéticas de repouso, que é da ordem de $2 \%$ a cada década. A cessação da função ovariana provoca redução do metabolismo, da quantidade de massa magra, e do gasto energético no exercício, além de estimular o acúmulo de gordura no tecido adiposo, contribuindo para o maior risco de obesidade e doença cardiovascular em mulheres após a menopausa. 3

Além do aumento no peso corporal total (obesidade global), a menopausa tem sido associada a um maior acúmulo de gordura no abdômen (obesidade abdominal). Inúmeros estudos relatam a influência da transição menopausal nas mudanças desfavoráveis na distribuição de gordura corporal, contribuindo para explicar o maior risco cardiovascular em mulheres $3-5$ nessa fase da vida.

A Sociedade Brasileira de Cardiologia enfatiza a nítida correlação entre a obesidade e o risco de DCV, 6 principal causa de morte hospitalar entre as brasileiras. ${ }^{7} \mathrm{Na}$ região metropolitana de São Paulo, em 2006, as DCV foram responsáveis por $22,1 \%$ das mortes em mulheres de 40 a 49 anos; $25,1 \%$ em mulheres de 50 a 59 anos e $26,9 \%$ em mulheres de 60 a 69 anos. Nesse mesmo ano, 12,2\% das internações de mulheres de 40 a 69 anos no Sistema Único de Saúde (SUS) foram atribuídas a essas causas. ${ }^{7}$

A deficiência estrogênica e as mudanças desfvoráveis no estilo de vida, principalmente em relação à qualidade da dieta e ao sedentarismo, vêm sendo mais conclusivamente apontadas como responsáveis pelo atual perfil de morbi-mortalidade em mulheres após os 50 anos nos Estados Unidos. ${ }^{8}$ No Brasil, embora os dados de morbidade sejam escassos, os dados de mortalidade em mulheres após a menopausa justificam a realização de mais estudos nesta área, com o intuito de fornecer subsídios para a promoção à saúde e prevenção de doenças, melhorando a qualidade de vida em idades avançadas.

O presente estudo teve como objetivo verificar a prevalência de obesidade global e obesidade abdominal e sua associação ao grau de instrução, nível de atividade física, paridade e terapia hormonal da menopausa (THM) em um grupo de mulheres na pós-menopausa.

\section{Métodos}

Em estudo transversal, com coleta de dados primários, foram avaliadas 157 mulheres na pósmenopausa (sem menstruação há pelo menos 12 meses), atendidas no Ambulatório de Saúde da Mulher no Climatério (ASMUC) do Centro de Saúde Escola "Geraldo de Paula Souza" da Faculdade de Saúde Pública (FSP) da Universidade de São Paulo, (USP) no período de abril a setembro de 2002, e no Ambulatório de Ginecologia do Hospital Universitário HU) da Universidade de São Paulo, na cidade de São Paulo, Brasil, no período de janeiro a junho de 2006. Consideraram-se critérios para inclusão na pesquisa a inscrição nos serviços citados, ausência de qualquer orientação dietética prévia nesses serviços e ausência de diagnóstico prévio de doença cardiovascular. O projeto de pesquisa foi aprovado pelo Comitê de Ética em Pesquisa da Faculdade de Saúde Pública da Universidade de São Paulo e pelo Comitê de Ética em Pesquisa do Hospital Universitário da Universidade de São Paulo.

As variáveis estudadas foram:

- Idade: calculada em anos completos na data da entrevista.

- Idade na menopausa: definida como a idade na última menstruação referida pela entrevistada;

- Grau de instrução: de acordo com a última série estudada, categorizado em até sete anos de estudo ou oito anos ou mais anos de estudo;

- Nível de atividade física; determinado conforme o Questionário Internacional de Atividade Física (International Physical Activity Questionnaire-IPAQ). ${ }^{9}$ As mulheres foram classificadas nas seguintes categorias: sedentária, insuficientemente ativa, ativa e muito ativa;

- Terapia hormonal da menopausa: as mulheres foram questionadas quanto ao uso atual ou pregresso de hormônios, bem como ao tipo e tempo de uso e foram classificadas em dois grupos: as que nunca tinham utilizado THM ou relataram uso pregresso inferior a 12 meses (Sem THM) e as usuárias por via oral, por pelo menos 12 meses (Com THM);

- Paridade: foram classificadas em uma das seguintes categorias: 0,1 ou 2 partos; 3 ou mais partos.

- Obesidade global verificada por meio de dois métodos: índice de massa corporal (IMC=peso/ estatura 2 ), adotando-se a classificação da World 
Health Organization: 10 não obesa $\left(<30,0 \mathrm{~kg} / \mathrm{m}^{2}\right)$ e obesa $\left(\geq 30,0 \mathrm{~kg} / \mathrm{m}^{2}\right)$ e percentual de gordura corporal (\%GC), obtido pelo método de bioimpedância elétrica, utilizando-se aparelho portátil RJL-SYSTEMS e adotada a seguinte classificação: 11 não obesa $(<37 \%)$ e obesa $(\geq 37 \%)$;

- Obesidade abdominal: obtida por meio da relação entre as medidas da circunferência da cintura (mensurada no ponto médio entre a última costela e a crista ilíaca) e do quadril (mensurado acima no trocânter ou na região de maior circunferência) tendo sido considerado como ponto de corte a RCQ de 0,85: 10 não obesa $(<0,85)$, obesa $(\geq 0,85)$.

Para a análise dos dados, foi criado um banco de dados no software Epi-Info 6.0. As associações entre a prevalência de obesidade global e obesidade abdominal e o grau de instrução, nível de atividade física, terapia hormonal da menopausa e paridade, foram comparadas pelo teste qui-quadrado com o programa Stata 7.0. Em todas as análises, adotou-se o nível de significância $\alpha=5 \%$.

\section{Resultados}

A média etária da população foi 56,1 anos, enquanto que a média de idade na menopausa foi 47,6 anos. No entanto, ao se considerar o tipo de menopausa, constatou-se que as mulheres com menopausa natural atingiram-na, em média, aos 48,4 anos e aquelas que foram submetidas a ooforectomia bilateral (menopausa cirúrgica) atingiram-na, em média, aos 43,0 anos. Grande parte $(47,8 \%)$ declarou ter de um a sete anos de estudo; $52,3 \%$ foram classificadas como sedentárias ou insuficientemente ativas e $47,1 \%$ referiram nunca ter feito THM. Quanto à paridade, $69,4 \%$ declararam ter tido pelo menos três partos. (Tabela 1)

O IMC médio das mulheres do estudo foi 28,0 $\mathrm{kg} / \mathrm{m}^{2}$. Segundo esse critério, 10 (Figura 1) a maioria tinha algum grau de excesso de peso, sendo $35,0 \%$ risco de obesidade e $34,4 \%$ obesidade, destas $26,1 \%$ eram classe I, 5,7\% classe II e 2,6\% classe III. O percentual médio de gordura corporal foi $34,9 \%$. De acordo com o $\% \mathrm{GC}, 40,1 \%$ das mulheres foram consideradas obesas. A diferença entre esta proporção de obesas e aquela detectada pelo IMC $(34,4 \%)$ não foi estatisticamente significativa $(p=0,350)$.

Houve associação estatisticamente significativa da prevalência de obesidade global, segundo o IMC, com o grau de instrução $(p=0,006)$, a prática de atividade física $(p<0,001)$, o uso de terapia hormonal da menopausa $(p=0,007)$ e a paridade $(p=0,002)$
Tabela 1

Distribuição das mulheres na pós-menopausa atendidas em dois ambulatórios segundo grau de instrução, nível de atividade física, uso de terapia hormonal da menopausa e paridade. São Paulo, Capital, 2002 e 2006.

\begin{tabular}{llrr}
\hline Variável & Categorias & N & $\%$ \\
\hline Grau & & & \\
de instrução & Sem instrução & 9 & 5,7 \\
& De um a sete anos de estudo & 75 & 47,8 \\
& Oito anos ou mais de estudo & 73 & 46,5 \\
& & & \\
Nível de & & 18 & 11,5 \\
$\quad$ atividade física & Sedentária & 64 & 40,8 \\
& Insuficientemente ativa & 70 & 44,6 \\
& Ativa & 5 & 3,2 \\
& Muito ativa & & \\
Terapia & & 74 & 47,1 \\
$\quad$ hormonal & Nunca usou & 39 & 24,9 \\
da menopausa & Já usou ou usa há menos de 12 meses & 44 & 28,0 \\
& Usa há mais de 12 meses & & \\
Paridade & & 48 & 30,6 \\
& Nulípara, um ou dois partos & 109 & 69,4 \\
\hline
\end{tabular}

Figura 1

Distribuição das mulheres na pós-menopausa atendidas em dois ambulatórios segundo obesidade global (índice de massa corporal e percentual de gordura corporal) e obesidade abdominal (relação cintura quadril). São Paulo, Capital, 2002 e 2006.

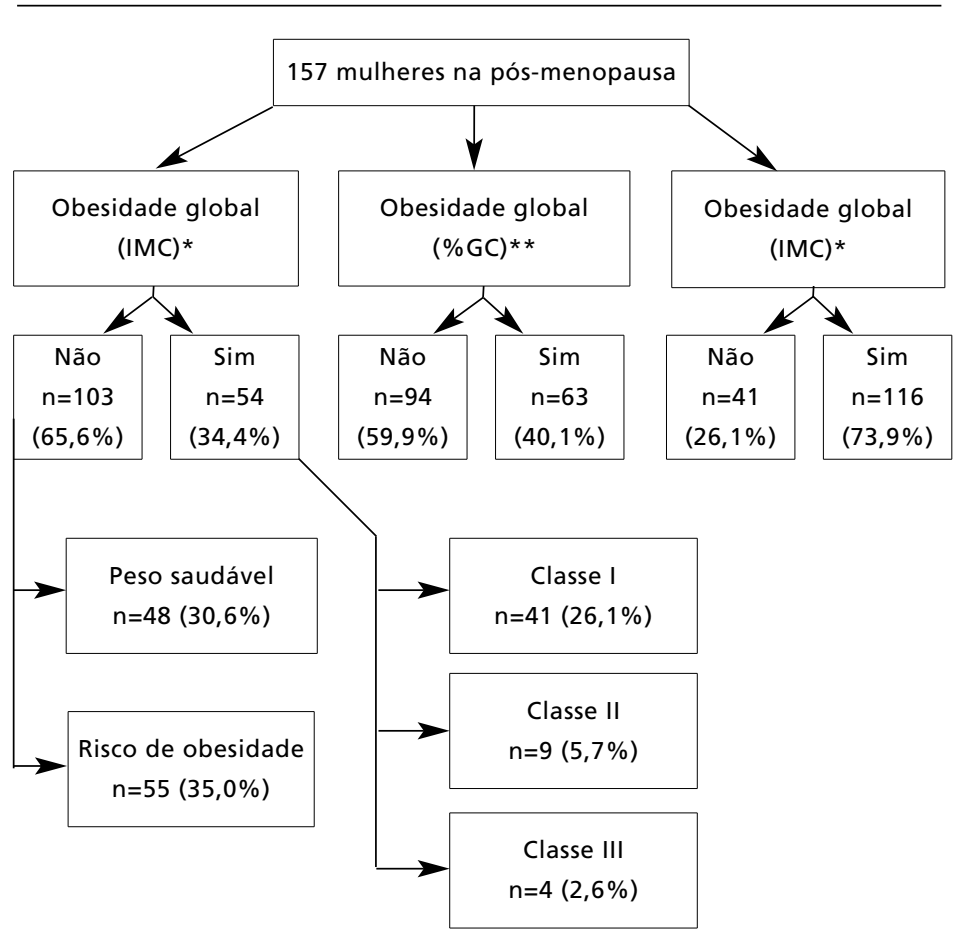


Prevalência de obesidade global, segundo índice de massa corporal (IMC), em mulheres na pós-menopausa atendidas em dois ambulatórios, de acordo com o grau de instrução, nível de atividade física, terapia hormonal da menopausa e paridade. São Paulo, Capital, 2002 e 2006.

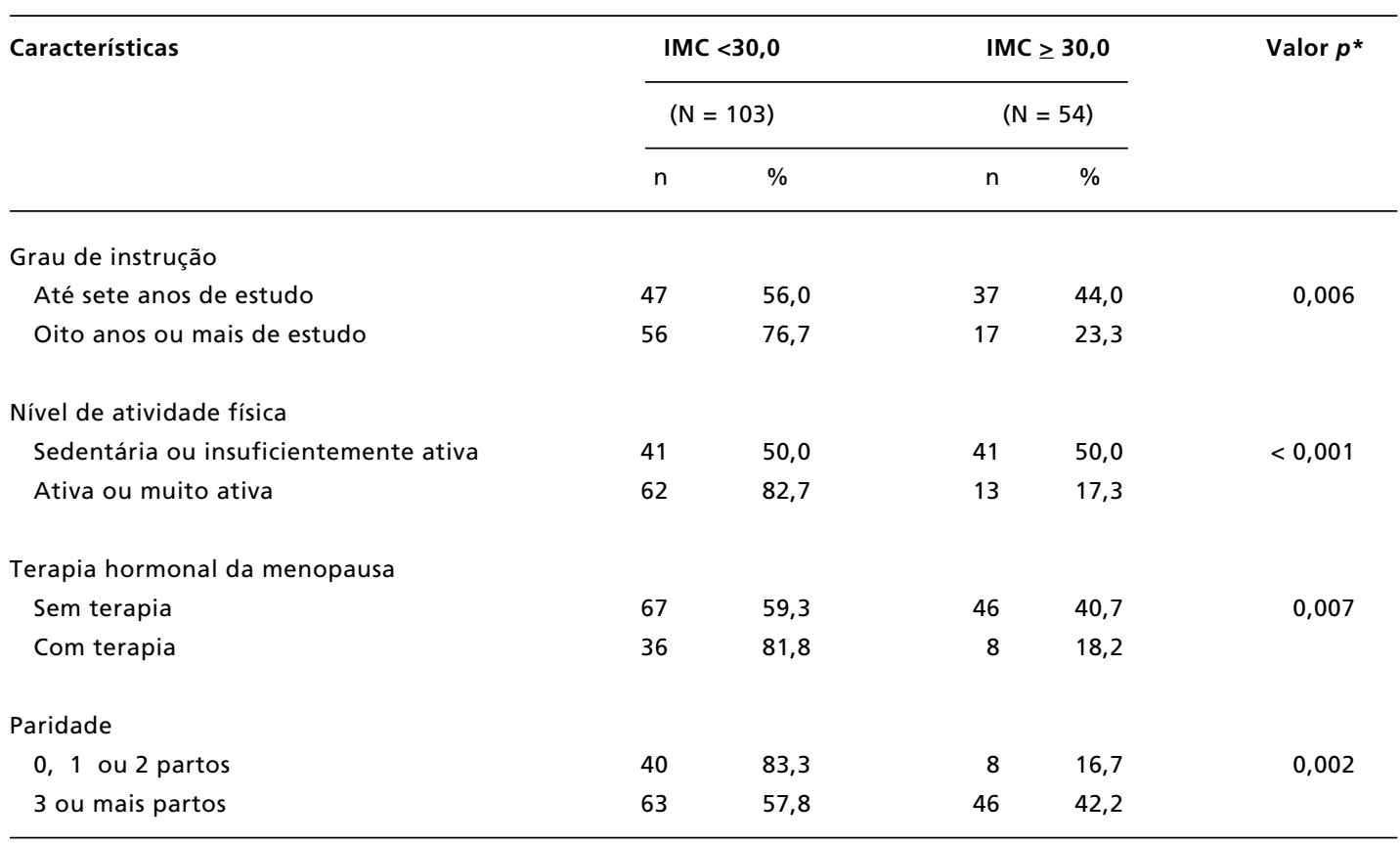

* Qui-quadrado (Pearson).

\section{Tabela 3}

Prevalência de obesidade global, segundo o percentual de gordura corporal (\%GC), em mulheres na pós-menopausa atendidas em dois ambulatórios, de acordo com o grau de instrução, nível de atividade física, terapia hormonal da menopausa e paridade. São Paulo, Capital, 2002 e 2006.

\begin{tabular}{|c|c|c|c|c|c|}
\hline \multirow[t]{3}{*}{ Características } & \multirow{2}{*}{\multicolumn{2}{|c|}{$\begin{array}{c}\% G C<37 \% \\
(N=94)\end{array}$}} & \multirow{2}{*}{\multicolumn{2}{|c|}{$\frac{\% G C \geq 37 \%}{(N=63)}$}} & \multirow[t]{3}{*}{ Valor $p^{*}$} \\
\hline & & & & & \\
\hline & $\mathrm{n}$ & $\%$ & $\mathrm{n}$ & $\%$ & \\
\hline \multicolumn{6}{|l|}{ Grau de instrução } \\
\hline Até sete anos de estudo & 49 & 58,3 & 35 & 41,7 & 0,673 \\
\hline Oito anos ou mais de estudo & 45 & 61,6 & 28 & 38,4 & \\
\hline \multicolumn{6}{|l|}{ Nível de atividade física } \\
\hline Sedentária ou insuficientemente ativa & 39 & 47,6 & 43 & 52,4 & $<0,001$ \\
\hline Ativa ou muito ativa & 55 & 73,3 & 20 & 26,7 & \\
\hline \multicolumn{6}{|l|}{ Terapia hormonal da menopausa } \\
\hline Sem terapia & 62 & 54,9 & 51 & 45,1 & 0,040 \\
\hline Com terapia & 32 & 72,7 & 12 & 27,3 & \\
\hline \multicolumn{6}{|l|}{ Paridade } \\
\hline 0,1 ou 2 partos & 34 & 70,8 & 14 & 29,2 & 0,063 \\
\hline 3 ou mais partos & 60 & 55,0 & 19 & 45,0 & \\
\hline
\end{tabular}

* Qui-quadrado (Pearson). 
Prevalência de obesidade abdominal, segundo relação cintura quadril (RCQ), em mulheres na pós-menopausa atendidas em dois ambulatórios, de acordo com o grau de instrução, nível de atividade física, terapia hormonal da menopausa e paridade. São Paulo, Capital, 2002 e 2006.

\begin{tabular}{|c|c|c|c|c|c|}
\hline \multirow[t]{3}{*}{ Características } & \multirow{2}{*}{\multicolumn{2}{|c|}{$\begin{array}{c}\mathrm{RCQ}<0,85 \\
(\mathrm{~N}=41)\end{array}$}} & \multirow{2}{*}{\multicolumn{2}{|c|}{$\begin{array}{c}\mathrm{RCQ} \geq 0,85 \\
(\mathrm{~N}=116)\end{array}$}} & \multirow[t]{3}{*}{ Valor $p^{*}$} \\
\hline & & & & & \\
\hline & $\mathrm{n}$ & $\%$ & $\mathrm{n}$ & $\%$ & \\
\hline \multicolumn{6}{|l|}{ Grau de instrução } \\
\hline Até sete anos de estudo & 16 & 19,0 & 68 & 81,0 & 0,030 \\
\hline Oito anos ou mais de estudo & 25 & 34,2 & 48 & 65,8 & \\
\hline \multicolumn{6}{|l|}{ Nível de atividade física } \\
\hline Sedentária ou insuficientemente ativa & 17 & 20,7 & 65 & 79,3 & 0,108 \\
\hline Ativa ou muito ativa & 24 & 32,0 & 51 & 68,0 & \\
\hline \multicolumn{6}{|l|}{ Terapia hormonal da menopausa } \\
\hline Sem terapia & 27 & 23,9 & 86 & 76,1 & 0,310 \\
\hline Com terapia & 14 & 31,8 & 30 & 68,2 & \\
\hline \multicolumn{6}{|l|}{ Paridade } \\
\hline 0,1 ou 2 partos & 14 & 29,2 & 34 & 70,8 & 0,563 \\
\hline 3 ou mais partos & 27 & 24,8 & 82 & 75,2 & \\
\hline
\end{tabular}

* Qui-quadrado (Pearson).

(Tabela 2), constatando-se maior proporção de mulheres obesas naquelas com até sete anos de estudo, não sedentárias ou insuficientemente ativas, nas sem THM e nas com três partos ou mais.

Contudo, a prevalência de obesidade global, segundo a \%GC, mostrou-se associada ao nível de atividade física $(p<0,001)$ e ao uso de terapia hormonal da menopausa $(p=0,040)$ nesse grupo de mulheres, mas não ao grau de instrução e nem à paridade. (Tabela 3)

A média da RCQ foi de 0,90 em todas as mulheres na pós-menopausa, a grande maioria $(73,8 \%)$ tendo apresentado obesidade abdominal. (Figura 1)

A proporção de mulheres com obesidade abdominal foi maior entre aquelas com até sete anos de estudo ( $p=0,030)$, entre as sedentárias ou insuficientemente ativas, no grupo "Sem THM" e naquelas que tiveram três ou mais partos; não ouve diferença significante. (Tabela 4)

\section{Discussão}

No Brasil, a escassez de estudos de base populacional em mulheres na menopausa impossibilita a comparação nacional. Na cidade de São Paulo, em estudo com 1400 mulheres, a média etária ao atingir a menopausa natural foi 48,5 anos, 12 muito semelhante à encontrada no presente estudo. Em Campinas, São Paulo, um inquérito populacional domiciliar com 465 mulheres de 45 a 60 anos, 13 mostrou que a média de idade da instalação da menopausa natural foi 2,8 anos maior do que a encontrada neste estudo ou seja, 51,2 anos, porém semelhante à encontrada nos Estados Unidos.

A prevalência de obesidade global foi alta no grupo de mulheres estudado. A diferença na prevalência de obesidade global observada ao utilizar diferentes métodos (IMC e \%GC) não foi significativa.

A tendência na elevação das taxas de obesidade na população feminina, mesmo em países em desenvolvimento como o Brasil, tem sido mostrada por alguns estudos. ${ }^{14,15}$ Inquéritos realizados nas últimas três décadas, utilizando o índice de massa corporal (IMC) para avaliar o estado nutricional da população brasileira-o Estudo Nacional da Despesa Familiar, em 1975,14 a Pesquisa Nacional sobre Demografia e Saúde, em 1996,14 e a Pesquisa de Orçamentos Familiares, em 2002 e 200315 - constataram substancial aumento na prevalência de obesidade no grupo 
de mulheres brasileiras adultas: 5,3\% em 1975; $10,2 \%$ em 1996 e $13,1 \%$ em 2002-2003; taxas que são ainda maiores nas mulheres acima dos 40 anos. No município de Cotia, região metropolitana de São Paulo, a prevalência de obesidade entre mulheres de 40 a 59 anos foi de 20,3\%, entre 1990 e $1991 .{ }^{16} \mathrm{Em}$ 2002, em estudo com 200 pacientes de um ambulatório público da cidade de São Paulo, foi de 24,6\% em mulheres de 40 a 49 anos e $39,0 \%$ em mulheres de 50 a 59 anos. ${ }^{17}$

Mais da metade das mulheres do presente estudo era sedentária ou insuficientemente ativa, prevalência ainda maior do que a encontrada em estudo com 632 mulheres de 30 a 69 anos, em 29 cidades do estado de São Paulo (45,6\%), 18 e estas apresentaram maior prevalência de obesidade global.

A diminuição no nível de atividade física foi o maior preditor do ganho de peso em 1064 mulheres, em estudo longitudinal desenvolvido na Escócia entre 1991 e 1993.19 Mulheres na pré-menopausa, que praticavam atividade moderada cinco vezes na semana ou atividade vigorosa por três vezes na semana, apresentaram IMC e \%GC significativamente menores do que aquelas que não praticavam atividade física regular. 20 Este vem sendo apontado como o principal fator modificável do estilo de vida, associado à redução da morbidade e mortalidade associada à obesidade em mulheres. 21

Os efeitos benéficos da atividade física para a promoção à saúde e prevenção de agravos são inúmeros, principalmente no tocante à doença cardiovascular, com evidências mostrando diminuição da pressão arterial, dos níveis séricos de LDL-colesterol triglicérides e inibição da agregação plaquetária. 22

A maior parte das mulheres estudadas utilizavam ou já tinham utilizado algum tipo de terapia hormonal. Em Campinas, em um inquérito populacional domiciliar realizado no ano de 1997 , observou-se que $48,6 \%$ das mulheres na pós-menopausa utilizavam ou já tinham utilizado esta terapia. ${ }^{23} \mathrm{~A}$ alta proporção de uso de THM provavelmente decorreu da divulgação dos resultados de estudos sobre os benefícios da terapia hormonal, destacandose a redução no risco de DCV na mulher após a menopausa. ${ }^{24}$ Contudo, recente estudo americano 25 recomendou cautela quanto ao posicionamento sobre os benefícios da THM, anteriormente consolidados. Em mulheres saudáveis na pós-menopausa, os resultados apontaram que os riscos à saúde excederam os benefícios do uso: o risco de fraturas do quadril e de câncer colo-retal diminuiu, porém o risco de câncer de mama, infarto do miocárdio, acidente vascular cerebral e tromboembolismo venoso aumentou.
No presente estudo, encontrou-se proporção menor de mulheres com obesidade global no grupo de usuárias de THM oral por pelo menos 12 meses, achados corroborados por outros estudos, que concluíram não haver evidências de que a reposição estrogênica propicie o aumento de peso em mulheres na pós-menopausa, ${ }^{19,26}$ ou que a THM poderia prevenir o aumento de peso nessa fase da vida. 27 Entretanto, por ser este estudo de caráter transversal, nada se pode inferir quanto ao possível papel da THM e o excesso de peso e gordura corporal, pelo fato de não se conhecer o estado nutricional das mulheres antes do início da terapia hormonal.

Os estudos enfocando THM e mudanças corporais na mulher mostram resultados bastante controversos, provavelmente em decorrência de inúmeros fatores que podem relacionar-se ao aumento de peso, como alimentação inadequada, atividade física insuficiente, maior número de partos, consumo de bebidas alcoólicas e o próprio processo de envelhecimento. 28,29

As mulheres na pós-menopausa que tinham menor grau de instrução e maior paridade, também apresentaram maior prevalência de obesidade global segundo o IMC. O censo de 2000 revelou que 72,3\% das mulheres brasileiras de 40 anos ou mais apresentavam nível de instrução baixo (22,2\% sem instrução e $50,1 \%$ com um a sete anos de estudo), 30 proporção ainda maior do que a encontrada neste estudo. Esse é um fator fundamental, considerando-se sua associação ao risco de doenças: mulheres com menor nível educacional apresentam maior risco para fatores associados à doença cardiovascular e diabetes tipo II, caracterizando a síndrome plurimetabólica. 31,32 Em estudo com mulheres chinesas, o nível educacional e a ocupação associaram-se inversamente à pressão arterial sistólica e à diastólica, ao IMC e ao número de cigarros consumidos ao dia, em análises ajustadas pela idade; e este foi enfatizado como o fator mais importante, dentre os indicadores socioeconômicos, relacionado ao risco cardiovascular. ${ }^{33}$

A paridade está positivamente associada ao aumento de peso e obesidade em países desenvolvidos e isto explica-se pelo excesso de aumento de peso no período gestacional. Nos países em desenvolvimento, nos quais o número maior de gestações por mulher é acompanhado de um tempo menor entre as gestações, essa relação é ainda mais expressiva. ${ }^{34}$ Mesmo em países desenvolvidos como a Finlândia, a média de aumento de peso durante a gestação aumentou no período de 1960 a 2000, o que pode explicar a maior prevalência de obesidade em idades mais avançadas. 35 
No tocante à obesidade abdominal, a relação cintura-quadril revelou que a proporção de mulheres na pós-menopausa com risco para doenças cardiovasculares foi muito alta e apenas o grau de instrução associou-se à RCQ.

Para o diagnóstico de obesidade abdominal, optou-se pela RCQ fundamentando-se nos resultados de outros estudos, 36,37 entre os quais destaca-se o Iowa Women's Health Study com uma coorte de 31.702 mulheres com idade de 55 a 69 anos, que mostrou a RCQ como melhor preditor antropométrico da mortalidade total, comparada ao IMC e à circunferência da cintura (CC). Esses autores concluíram que a RCQ oferece melhor informação prognóstica do que os outros parâmetros, já que associou-se positivamente à mortalidade por doença arterial coronariana, outras doenças cardiovasculares, câncer e outras causas, enquanto todos os indicadores antropométricos (RCQ, IMC e CC) associaram-se apenas à incidência de diabetes e hipertensão. 36

De fato a menopausa tem sido associada a maior acúmulo de gordura no abdômen e, conseqüentemente, ao maior risco cardiovascular em mulheres com mais de 50 anos.4,5 Nascimento 38 observou que, em mulheres entre 45 a 65 anos, a RCQ correlacionou-se positivamente a importantes fatores de risco para DCV, como o colesterol total $(\mathrm{r}=0,31$; $p=<0,001)$ e LDL-colesterol $(\mathrm{r}=0,28 ; p<0,001)$ e negativamente ao HDL-colesterol $(\mathrm{r}=-0,17 ; p=0,00)$, sabidamente um fator protetor contra aterogênese.

Outros estudos no Brasil constataram altas proporções de mulheres com distribuição de gordura corporal andróide. No município do Rio de Janeiro, Pereira et al. 39 encontraram obesidade abdominal em mais de $70 \%$ das mulheres estudadas, especialmente

\section{Referências}

1. NIH (National Institutes of Health), NHLBI (National Heart, Lung and Blood Institute) NAASO (North American Association for the Study of Obesity). Clinical guidelines on the identification, evaluation and treatment of overweight and obesity in adults: executive summary. Am J Clin Nutr. 1998; 68: 899-917.

2. WHO (World Health Organization). Global database on obesity and body mass index (BMI) in adults. Nutrition data banks. Available from: http:/www.who.int/nut/ db_bmi.htm http://www.who.int/nut/db_bmi.htm. [2001 Jul 23]

3. Poehlman ET, Tchernof A. Transversing the menopause: changes in energy expenditure and body composition. Coron Artery Dis. 1998; 9: 799-803. naquelas com idade acima de 45 anos. Fornés et al. 40 por sua vez, detectaram que $72,5 \%$ das 615 mulheres com 20 anos ou mais residentes em Cotia, São Paulo, exibiam obesidade abdominal.

Assim como nesse estudo, Kac et al.41 concluíram que, em mulheres de 16 a 45 anos, maior escolaridade significou menor RCQ. No estudo de Machado e Sichieri42 a RCQ associou-se positivamente à idade, tabagismo e índice de massa corporal; e inversamente à escolaridade, renda e atividade física, mostrando que estilo de vida e indicadores sociais associam-se a alterações na RCQ e, portanto, ao risco de complicações metabólicas relacionadas à incidência de doenças cardiovasculares.

\section{Conclusões}

O presente estudo mostrou que a prevalência de obesidade global, obesidade abdominal e o conseqüente risco de complicações metabólicas e DCV são aspectos preocupantes em relação à saúde de mulheres após a menopausa.

As mulheres após a menopausa, usuárias de THM e com nível de atividade física alto e muito alto, exibiram menor prevalência de obesidade global. Contudo, a prevalência de obesidade abdominal foi alta, independentemente do uso de THM oral durante pelo menos 12 meses e maior ainda nas mulheres com menos anos de estudo.

Com base nesses resultados, conclui-se que a atenção multidisciplinar à saúde da mulher, principalmente na pré-menopausa, é imprescindível para a prevenção das doenças que mais acometem as mulheres e são as principais causas de mortalidade nesse grupo.

4. Toth MJ, Tchernof A, Sites CK, Poehlman ET. Effect of menopausal status on body composition and abdominal fat distribution. Int J Obes Relat Metab Disord. 2000; 24: 22631 .

5. Gambacciani M, Ciaponi M, Cappagli B, De Simone L, Orlandi R, Genazzani AR. Prospective evaluation of body weight and body fat distribution in early post-menopausal women with and without hormonal replacement therapy. Maturitas. 2001; 39: 125-32.

6. Santos RD, Timerman S, Sposito AC, coordenadores. Diretrizes para cardiologistas sobre excesso de peso e doença cardiovascular dos Departamentos de Aterosclerose, Cardiologia Clínica e FUNCOR da Sociedade Brasileira de Cardiologia. Arq Bras Cardiol. 2002; 78 (Supl.1): 1-13 
7. Brasil. Ministério da Saúde. Sistema de informações hospitalares do SUS. Mortalidade hospitalar do SUS-por local de residência-Brasil. Disponível em: http//www.tabnet. datasus.gov.br [2007 jul 17].

8. NAMS (North American Menopause Society). Menopause core curriculum study guide. Cleveland, $\mathrm{OH}$ : The North American Menopause Society; 2002.

9. Mader U, Martin BW, Schutz Y, Marti B. Validity of four short physical activity questionnaires in middle-aged persons. Med Sci Sports Exerc. 2006; 38: 1255-66.

10. WHO (World Health Organization). Obesity: preventing and managing the global epidemic: report of a WHO Consultation on Obesity. Geneva; 1997.

11. Zhu S, Wang Z, Shen W, Heymsfield SB, Heshka S. Percentage body fat ranges associated with metabolic syndrome risk: results based on the third National Health and Nutrition Examination Survey (1988-1994). Am J Clin Nutr. 2003; 78: 228-35.

12. Aldrighi JM, Alecrin IN, Oliveira PR, Shinomata HO. Tabagismo e antecipação da idade na menopausa. Rev Assoc Med Bras. 2005; 51: 51-3.

13. Pedro AO, Pinto Neto AM, Paiva LHSC, Osis MJ, Hardy E. Idade de ocorrência da menopausa natural em mulheres brasileiras: resultados de um inquérito populacional domiciliar. Cad Saúde Pública. 2003; 19: 17-25.

14. Monteiro CA. Epidemiologia da obesidade. In: Halpern A, Mattos AFG, Suplicy H, Mancini M, Zanello MT. Obesidade. São Paulo: Lemos; 1998.

15. IBGE (Instituto Brasileiro de Geografia e Estatística). Diretoria de Pesquisas. Coordenação de Índices e Preços. Pesquisa de orçamentos familiares 2002-2003. Rio de Janeiro; 2004.

16. Martins IS, Velasquez-Melendez G, Cervato AM. Estado nutricional de grupamentos sociais da área metropolitana de São Paulo, Brasil. Cad Saúde Pública. 1999; 15: 41-78.

17. França AP. Estado nutricional e risco de doença cardiovascular de mulheres no climatério atendidas em um ambulatório da cidade de São Paulo [dissertação mestrado]. São Paulo: Faculdade de Ciências Farmacêuticas, Faculdade de Economia e Administração, Faculdade de Saúde Pública da Universidade de São Paulo; 2003.

18. Matsudo SM, Matsudo VR, Araújo T, Andrade D, Andrade E, Oliveira L, Braggion G. Nível de atividade física da população do estado de São Paulo: análise de acordo com o gênero, idade, nível socioeconômico, distribuição geográfica e de conhecimento. Rev Bras Cien Mov. 2002; 10: 4150 .

19. Macdonald HM, New SA, Campbell MK, Reid DM. Longitudinal changes in weight in perimenopausal and early postmenopausal women: effects of dietary energy intake, energy expenditure, dietary calcium intake and hormone replacement therapy. Int J Obes. 2003; 27: 66976.

20. Holcomb CA, Heim DL, Loughin TM. Physical activity minimizes the association of body fatness with abdominal obesity in white, pre-menopausal women: results from the third national health and nutrition examination survey. J Am Dietet Assoc. 2004; 104: 1859-62.
21. Dubnov G, Brzezinski A, Berry EM. Weight control and the management of obesity after menopause: the role of physical activity. Maturitas. 2003; 44: 89-101.

22. CDC (Center for Disease Control and Prevention), NCCDPHP (National Center for Chronic Disease Prevention and Health Promotion), DNPA (Division of Nutrition and Physical Activity). Physical activity and health. A report of the surgeon general. Available from: http://www.cdc.gov/nccdphp/sgr/pdf/women.pdf> [2002 Apr 14]

23. Pinto Neto AM, Pedro AO, Hardy E, Osis MJD, CostaPaiva LHS, Martinez EZ. Caracterização das usuárias de terapia de reposição hormonal do Município de Campinas, São Paulo. Cad Saúde Pública. 2002; 18: 121-7.

24. Grant E. Sexual chemistry: understanding your hormones, the pill and HRT. Londres: Cedar; 1994.

25. WGWHII (Writing Group for the Women's Health Initiative Investigators). Risks and benefits of estrogen plus progestin in healthy postmenopausal women. JAMA. 2002; 288: 321 33.

26. Norman RJ, Flight TH, Rees MC. O estrogen and progestogen hormone replacement therapy for perimenopausal and post-menopausal women: weight and body fat distribution. Cochrane Database System Rev. 2003; AB001018.

27. Gambacciani M, Ciaponi M, Cappagli B, De Simone L, Orlandi R, Genazzani AR. Prospective evaluation of body weight and body fat distribution in early postmenopausal women with and without hormonal replacement therapy. Maturitas. 2001; 39: 125-32.

28. Matthews KA, Abrams B, Crawford S, Miles T, Neer R, Powell LH, Wesley D. BMI in mid-life women: relative influence of menopause, hormone use and ethnicity. Int $\mathrm{J}$ Obes. 2001; 25: 863-73.

29. Ozbey N, Sencer E, Molvalilar S, Orhan Y. Body fat distribution and cardiovascular disease risk factors in pre- and postmenopausal obese women with similar BMI. Endocr J. 2002; 49: 503-9.

30. IBGE (Instituto Brasileiro de Geografia e Estatística). Censo demográfico 2000. Disponível em: http://www. ibge.gov.br/sidra> [2002 abr 28].

31. Wamala SP, Lynch J, Horsten M, Mittleman MA, SchenkGustafsson K, Orth-Gomer K. Education and the metabolic syndrome in women. Diabetes Care. 1999; 22: 1999-2003.

32. Muller A. Education, income inequality, and mortality: a multiple regression analysis. BMJ. 2002; 324: 1-4.

33. Yu Z, Nissinen A, Vartiainen E, Song G, Guo Z, Zheng JT, Tian H. Associations between socioeconomic status and cardiovascular risk factors in an urban population in China. Bull World Health Organ. 2000; 78: 1296-305.

34. Kim SA, Stein AD, Martorell R. Country development and the association between parity and overweight. Int J Obes. 2007; 31:805-12.

35. Kinnunen TI, Luoto R, Gissler M, Hemminki E. Pregnancy weight gain from 1960 s to 2000 in Finland. Int J Obes. $2003 ; 27: 1572-7$. 
36. Folsom AR, Kushi LH, Anderson KE, Mink PJ, Olson JE, Hong C, Sellers TA, Lazovich D, Prineas RJ. Associations of general and abdominal obesity with multiple health outcomes in older women: the Iowa Women's Health Study. Arch Intern Med. 2000; 160: 2117-28.

37. Cabrera MAS, Wanjgarten M, Gebara OCE, Diament J. Relação do índice de massa corporal, da relação cinturaquadril e da circunferência abdominal com a mortalidade em mulheres idosas: seguimento de cinco anos. Cad Saúde Pública. 2005; 21: 767-75.

38. Nascimento ML. Relação cintura/quadril, níveis séricos de colesterol e frações e doenças crônico-degenerativas em adultos jovens e idosos [dissertação mestrado]. São Paulo: Faculdade de Saúde Pública da Universidade de São Paulo; 1999.

Recebido em 4 de outubro de 2006

Versão final apresentada em 19 de setembro de 2007

Aprovado em 22 de outubro de 2007
39. Pereira RA, Marins VMR, Sichieri R. Nutritional profile of adult people in the municipality of Rio de Janeiro, Brazil 1996. In: Abstract of XVI International Congress of Nutrition; 1997 Jul 27-Aug 1; Montréal, Canada. Montréal: International Union of Nutritional Sciences; 1997. p. 302.

40. Fornés NS, Martins IS, Hernan M, Velásquez-Meléndez G, Ascherio A. Food frequency consumption and lipoproteins serum levels in the population of an urban area, Brazil. Rev Saúde Pública. 2000; 34: 380-7.

41. Kac G, Velásquez-Meléndez G, Coelho MASC. Fatores associados à obesidade abdominal em mulheres em idade reprodutiva. Rev Saúde Pública. 2001; 35: 46-51.

42. Machado PA, Sichieri R. Relação cintura-quadril e fatores de dieta em adultos. Rev Saúde Pública. 2002; 36: 198-204. 